\title{
Multi-Objective Optimization for the m-PDPTW: Aggregation Method With Use of Genetic Algorithm and Lower Bounds
}

\author{
I. Harbaoui Dridi, R. Kammarti, M. Ksouri, P. Borne
}

\section{Imen Harbaoui Dridi}

LAGIS : Ecole Centrale de Lille, Villeneuve d'Ascq, France

LACS : Ecole Nationale des Ingénieurs de Tunis,

Tunis - Belvédère. Tunisie

E-mail: imenharbaoui@gmail.com

\author{
Ryan Kammarti, Mekki Ksouri \\ LACS : Ecole Nationale des Ingénieurs de Tunis, \\ Tunis - Belvédère. Tunisie \\ E-mail: kammarti.ryan@planet.tn, Mekki.Ksouri@insat.rnu.tn
}

\section{Pierre Borne}

LAGIS : Ecole Centrale de Lille,

Villeneuve d'Ascq, France

E-mail: p.borne@ec-lille.fr

\begin{abstract}
The PDPTW is an optimization vehicles routing problem which must meet requests for transport between suppliers and customers in purpose to satisfy precedence, capacity and time constraints. We present, in this paper, a genetic algorithm for multi-objective optimization of a multi pickup and delivery problem with time windows (m-PDPTW), based on aggregation method and lower bounds. We propose in this sense a brief literature review of the PDPTW, present our approach to give a satisfying solution to the m-PDPTW minimizing the compromise between total travel cost and total tardiness time. Keywords: PDPTW, multi-objective, aggregation method, lower bounds.
\end{abstract}

\section{Introduction}

The vehicle routing planning and traffic management are considered a major logistical challenge in terms of supply, inter-plant transport or distribution transport. [1] Many studies have been directed mainly towards solving the vehicle routing problem (VRP). It's an optimization vehicle routing problem to meet travel demands. Other researchers became interested on an important variant of VRP which is the PDPTW (Pickup and Delivery Problem with Time Windows) with capacity constraints on vehicles.

The PDPTW is divided into two categories: 1-PDPTW (single-vehicle) and m-PDPTW multivehicle).

In the m-PDPTW problem which we are interested in, we consider a vehicles fleet $V_{k}$ of capacity $Q_{k}$ and a set of goods to transport providers to different destinations. The goal is to provide a set of customers under certain constraints concerning vehicles and their capacity, precedence between nodes, and this by minimizing the compromise between the total travel cost and total tardiness time. In this paper we present a literature review of the PDPTW followed by the proposed approach for the optimization of pick-up and delivery problem with time window, using the genetic algorithms, aggregation method and lower bounds. 


\section{Litterature Review}

\subsection{Vehicle routing problem}

The Vehicle Routing Problem (VRP) represents a multi-goal combinatorial optimization problem which has been the subject of many works and variations in the literature [2] [3]. The theory of the VRP is formulated as follows: given a depot D and a set of customers orders $\mathrm{C}=$ $(\mathrm{c} 1, \ldots, \mathrm{Cn})$, to build a package routing, for a finite number of vehicles, beginning and ending at the depot. In this routing, a customer must be served only once by a single vehicle and vehicle capacity transport for a routing should not be exceeded [4] [5]. The Meta heuristics were also applied to solve the vehicle routing problem. Among these methods, we can include ant colony algorithms, which were used by Montamenni, $\mathrm{R}$ et al for the resolution of DVRP [6], and by Sorin C. Negulescu et al to solve the Vehicle Route Allocation Problem (VRAP) [7]. Kaoru Hirota et al have presented a computational intelligence approach to VRSDP (Vehicle Routing, Scheduling, and Dispatching Problems). The objective of the VRSDP is to produce a delivery schedule for a group of vehicles, with respect to multiple users, so that while satisfying constraints delivery cost corresponding to users' order is minimized [8]. Savelsbergh et al have shown that the VRP is a NP-hard problem [9].

\subsection{The PDPTW: Pickup and Delivery Problem with Time Windows}

The PDPTW is a variant of VRPTW where in addition to the existence of time constraints, this problem implies a set of customers and a set of suppliers geographically located. Every routing must also satisfy the precedence constraints to ensure that a customer should not be visited before his supplier. [10] A dynamic approach for resolving the 1-PDP without and with time windows was developed by Psaraftis, H.N considering objective function as a minimization weighting of the total travel time and the non-customer satisfaction [11]. Jih, W et al have developed an approach based on the hybrid genetic algorithms to solve the 1-PDPTW, aiming to minimize combination of the total cost and total waiting time. [12] Another genetic algorithm was developed by Velasco, $\mathrm{N}$ et al to solve the 1-PDP bi-objective in which the total travel time must be minimized while satisfying in priority the most urgent requests. In this literature, the method proposed to resolve this problem is based on a No dominated Sorting Algorithm (NSGAII). [13] Kammarti, $\mathrm{R}$ et al treat the 1-PDPTW, minimizing the compromise between the total travel distance, total waiting time and total tardiness time, using an evolutionary algorithm with special genetic operators, tabu search to provide a set of viable solutions. [14] [15]. This work has been extended, by proposing a new approach based on the use of lower bounds and Pareto dominance method, to minimize the compromise between the total travel distance, total waiting time and total tardiness time. [16] About the m-PDPTW, Sol, M et al have proposed a branch and price algorithm to solve the m-PDPTW, minimizing the vehicles number required to satisfy all travel demands and the total travel distance. [17] Quan, L et al have presented a construction heuristic based on the integration principle with the objective function, minimizing the total cost, including the vehicles fixed costs and travel expenses that are proportional to the travel distance. [18] A new metaheuristic based on a tabu algorithm, was developed by $\mathrm{Li}, \mathrm{H}$ et al to solve the m-PDPTW. [19] Li, H et al have developed a "Squeaky wheel" method to solve the m-PDPTW with a local search. [20] A genetic algorithm was developed by Harbaoui Dridi I et al treating the m-PDPTW to minimize the total travel distance and the total transport cost [21]. This work has been extended, by proposing a new approach based on the use of Pareto dominance method to give a set of satisfying solutions to the m-PDPTW minimizing total travel cost, total tardiness time and the vehicles number. [22] [23] 


\section{Mathematical Formulation}

Our problem is characterized by the following parameters:

- N: Set of customers, supplier and depot vertices,

- $N^{\prime}$ : Set of customers and supplier vertices,

- $N^{+}$: Set of supplier vertices,

- $N^{-}$: Set of customers vertices,

- $\mathrm{n}$ : Size of the initial population,

- K: Vehicle number,

- $d_{i j}$ : Euclidian distance between the vertex $\mathrm{i}$ and the vertex j. If $d_{i j}=\infty$ then the road between i and j doesn't exist,

- $t_{i j k}$ : Time used by the vehicle $\mathrm{k}$ to travel from the vertex I to the vertex $\mathrm{j}$,

- $\left[e_{i}, l_{i}\right]$ : Time window of the vertex $\mathrm{i}$,

- $s_{i}$ : Stopping time at the vertex i,

- $q_{i}$ : Goods quantity of the vertex i request. If $q_{i}>0$, the vertex i is a supplier; if $q_{i}<0$, the vertex $\mathrm{i}$ is a customer and if $q_{i}=0$ then the vertex was served.

- $Q_{k}$ : Capacity of vehicle $\mathrm{k}$,

- $\mathrm{i}=0 \ldots \mathrm{N}$ : Predecessor vertex index,

- $\mathrm{j}=0 \ldots \mathrm{N}:$ Successor vertex index,

- $\mathrm{k}: 1 \ldots \mathrm{K}$ : Vehicle index,

- $X i j k=\left\{\begin{array}{l}1 \text { If the vehicle travel from the vertex i to the vertex } \mathrm{j} \\ 0 \text { Else }\end{array}\right.$

- $A_{i}$ : Arrival time of the vehicle to the vertex i,

- $D_{i}$ : Departure time of the vehicle from the vertex i,

- $y_{i k}$ : The goods quantity in the vehicle $\mathrm{k}$ visiting the vertex $\mathrm{i}$,

- $C_{k}$ : Travel cost associated with vehicle $\mathrm{k}$,

- A vertex is served only once,

- A vertex is served only once,

- The capacity constraint must be respected,

- The depot is the starting and finishing vertex for the vehicle,

- The vehicle stops at every vertex for a period of time to allow the request processing,

- If the vehicle arrives at a vertex i before its time windows beginning date it waits. 
The function to minimize is given as follows:

$$
\text { Minimizef }=\left(\begin{array}{l}
\lambda_{1} c_{1} \sum_{k \in K} \sum_{i \in N} \sum_{j \in N} C_{k} d_{i j k} X_{i j k}+ \\
\lambda_{2} c_{2} \sum_{k \in K} \sum_{i \in N} \sum_{j \in N} \max \left(0, D_{i}-l_{i}\right) X_{i j k}
\end{array}\right)
$$

Where $\lambda_{i}$ and $c_{i}$ are weights and scaling coefficients.

Subject to:

$$
\begin{aligned}
& \sum_{i=1}^{N} \sum_{k=1}^{K} x_{i j k}=1, j=2, \ldots N \\
& \sum_{j=1}^{N} \sum_{k=1}^{K} x_{i j k}=1, i=2, \ldots N \\
& \sum_{i \in N} X_{i 0 k}=1, \forall k \in K \\
& \sum_{j \in N} X_{0 j k}=1, \forall k \in K \\
& \sum_{i \in N} X_{i u k}-\sum_{j \in N} X_{u j k}=0, \forall k \in K, \forall u \in N \\
& X_{i j k=1 \Rightarrow y_{j k}=y_{i k}+q_{i}, \forall i, j \in N ; \forall k \in K} \\
& y_{0 k=0, \forall k \in K} \\
& Q_{k} \geq y_{i k} \geq 0, \forall i \in N ; \forall k \in K \\
& D_{w} \leq D_{v}, \forall w \in N^{+} ; \forall v \in N^{-} \\
& D_{0}=0 \\
& X_{i j k}=1 \Rightarrow D_{i}+t_{i j k} \leq D_{j} \forall i, j \in N ; \forall k \in K
\end{aligned}
$$

The constraint (2) and (3) ensure that each vertex is visited only once by a single vehicle. The constraint (4) and (5) ensure that the vehicle routing is beginning and finishing in the depot. The constraint (6) ensures the routing continuity by a vehicle. (7), (8) and (9) are the capacity constraints. The precedence constraints are guaranteed by (10), (11) and (12).

\section{Genetic Algorithm For Optimization Of The m-PDPTW}

\subsection{Generation of the initial populations}

In our case, we generate two types of populations. A first population noted $P_{\text {node }}$ (Figure 1 ), which represents all nodes to visit with all vehicles, according to the permutation list coding. The second population noted $P_{\text {vehicle }}$ (Figure 2 ) indicates nodes number visited by each vehicle. 


\begin{tabular}{|l|l|l|l|l|l|l|l|l|l|l|l|l|}
\hline $\operatorname{Nods}(1)$ & 0 & 5 & 2 & 6 & 4 & 3 & 10 & 8 & 7 & 9 & 1 & 0 \\
\hline
\end{tabular}

Figure 1: The permutation list coding

\begin{tabular}{|c|c|c|c|c|}
\hline$V_{1}$ & $V_{2}$ & $V_{3}$ & $V_{4}$ & $V_{5}$ \\
\hline 6 & 4 & 0 & 0 & 0 \\
\hline
\end{tabular}

Figure 2: Example of Individual from the population $P_{\text {vehicle }}$

\subsection{Correction procedures}

Before beginning the construction of the population $\mathrm{P}_{\text {node/vehicule, we proceed to the correc- }}$ tion procedures of precedence and capacity between nodes. We consider the following couples customer / supplier: $(1,5),(2,8),(9,7),(10,3)$ and $(4,6)$, noting that $Q_{k \max }=60$ and $\mathrm{q}=20$, we present, respectively, in figures 3 and 4 the principle of correction precedence and capacity.

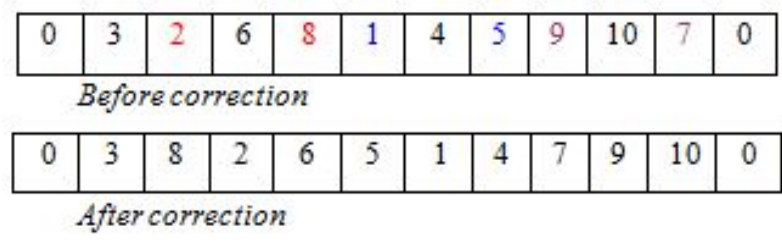

Figure 3: Correction precedence

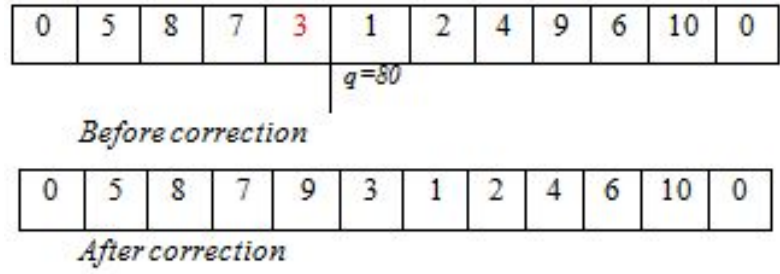

Figure 4: Correction capacity

\subsection{Computation procedure}

Taking into account the population $P_{\text {node }}$, correction procedures and $P_{\text {vehicle }}$ we illustrate in Figure 5 an example of an individual from the population $\mathrm{P}_{\text {node/vehicule }}$. Knowing that it is necessary to verify that a couple is visited by only one vehicle. [24]

with: $N^{\prime}=10$ and $\mathrm{K}=2$

Figure 6 represents the process to determine the population $\mathrm{P}_{\text {node/vehicule }}$.

The principles of different genetic operations such as crossover and mutation operator are detailed in our work [21]. 


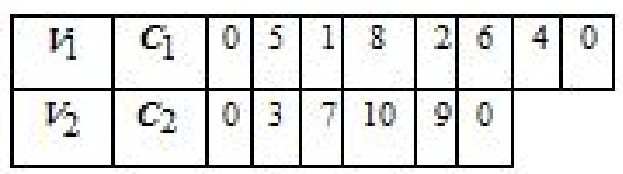

Figure 5: Example of an individual from the population $\mathrm{P}_{\text {node/vehicule }}$

\subsection{Multi-criteria evaluation}

A multi-objective problem is defined as an optimization vector problem, which seeks to optimize several components of a vector function cost.

\section{Pareto dominance method}

A multi-criteria problem $\mathrm{P}$ is composed of $\mathrm{n}$ variables, $\mathrm{m}$ inequality constraints, $\mathrm{p}$ equality constraints and $\mathrm{k}$ criteria that can be formulated as follows:

$$
\mathrm{P} \Rightarrow\left\{\begin{array}{l}
\min f(x)=\left[f_{1}, f_{2}, f_{3}, \ldots . . f_{k}(x)\right] \\
g_{i(x) \leq 0 \mathrm{i}=0 \ldots \mathrm{m}} \\
g_{j(x)=0 \mathrm{j}=0 \ldots \mathrm{p}}
\end{array}\right.
$$

However, it is necessary to find solutions representing a possible compromise between the criteria. The Pareto optimality concept introduced by the economist V. Pareto in the twentieth century is frequently used [25]. V. Pareto formulated the following concept: in a multi-criteria problem, there is equilibrium so that we can not improve one criterion without deteriorating at least one other. This equilibrium has been called Pareto optimal A solution is noted Pareto optimal if it is dominated by any other point in solutions space. These points are noted non dominated solutions.

A point $\mathrm{X} \in \mathrm{E}$ dominates $\mathrm{Y} \in \mathrm{E}$ if:

$$
\left\{\begin{array}{l}
\forall i, f_{i}(x) \leq f_{i}(y) \\
\text { and } \exists j, \operatorname{such} a s f_{j}(x)<f_{j}(y)
\end{array}\right.
$$

Figure 7 shows an example where we seek generations of the initial populations to minimize fland $\mathrm{f} 2$. The points 1,3 and 5 are not dominated. On the contrary point 2 is dominated by point 3 , and point 4 is dominated by point 5 .

\section{Aggregation method}

In the resolution of MOP (Multi objectives Problem), several traditional methods are transforming the MOP into a single objective problem. Among these methods we find the aggregation method. This is one of the first methods used to generate Pareto optimal solutions. It is to transform the problem (MOP) in a problem $\left(P M O_{\lambda}\right)$ which combines the different cost functions of the problem into a single objective function $\mathrm{F}$ generally linear [26]:

$$
F(x)=\sum_{i=1}^{n} c_{i} \lambda_{i} f_{i}(x)
$$

Where $\lambda_{i}$ and $c_{i}$ are weights and scaling coefficients, according to the application, that the different objectives are not necessarily commensurable. The constants $c_{i}$ are usually initialized to $\frac{1}{f_{i}(x *)}$ where $f_{i}(x *)$ is the optimal solution associated to the objective function $f_{i}$ considered 


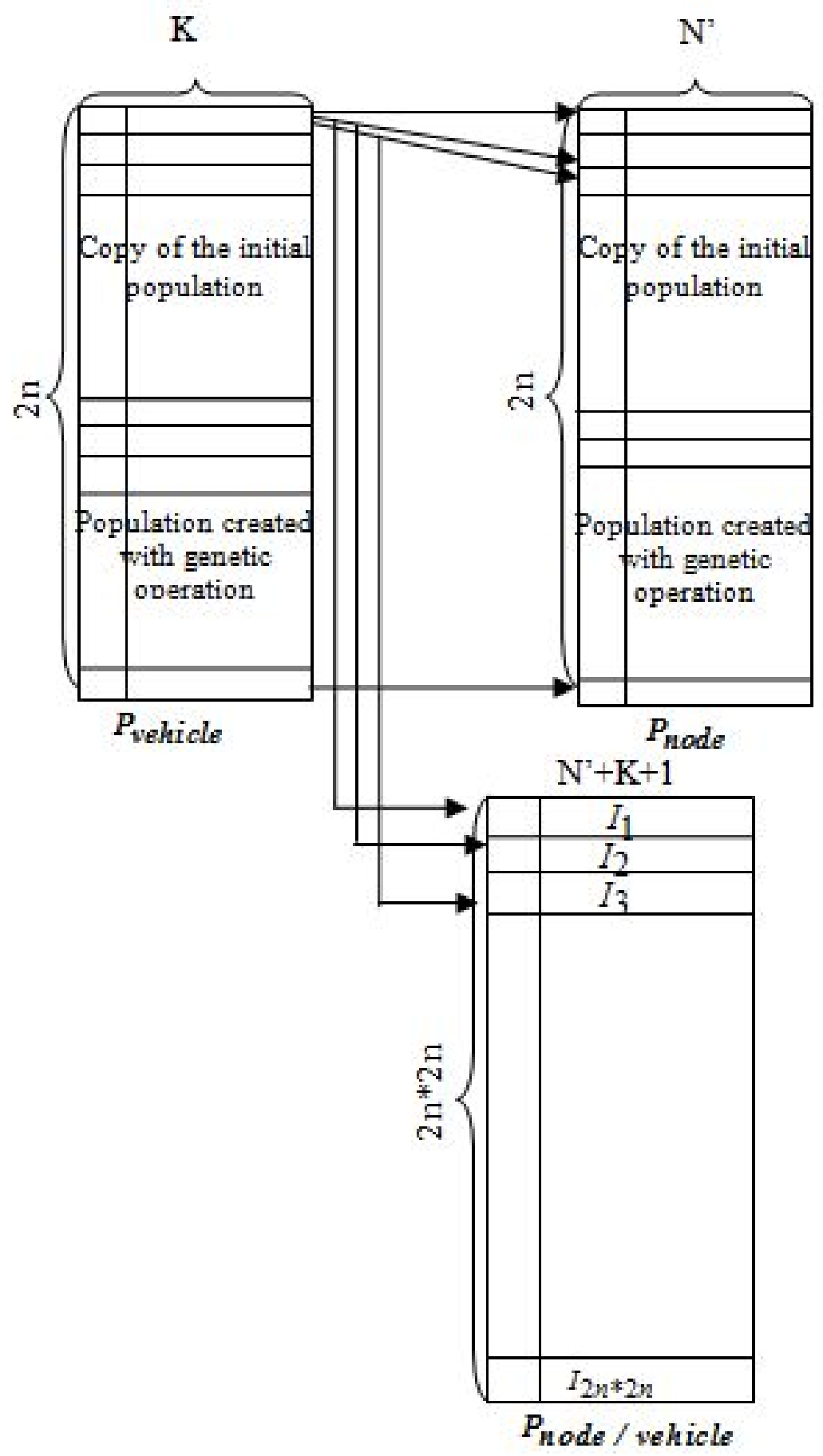

Figure 6: Computation procedure 


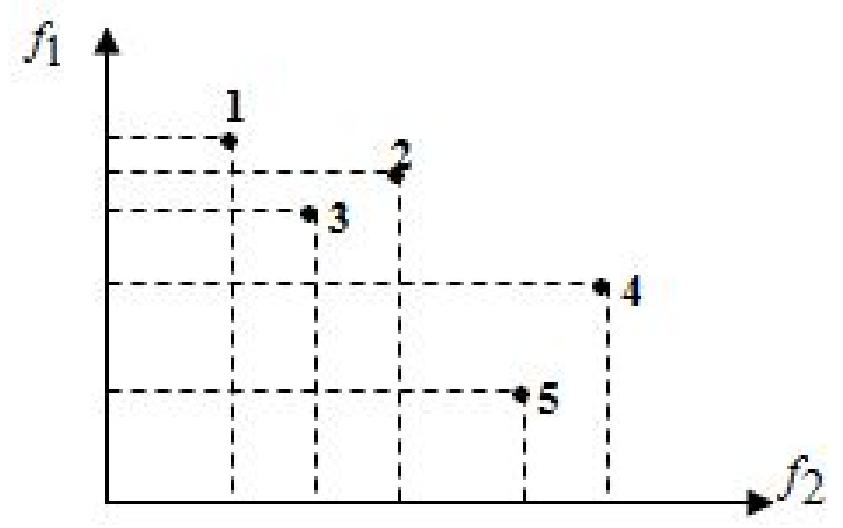

Figure 7: Dominance example

separately. The idea of the aggregation method (Figure 8) is to fix a weight vector i.e. find a hyper-plane in the objective space (a line for a bi-criteria problem) with a fixed orientation. The Pareto optimal solution is the point where the hyper-plane has a common tangent with a feasible space. The advantage of the aggregation method is to produce a single solution and thus do not require interaction with the decision maker.

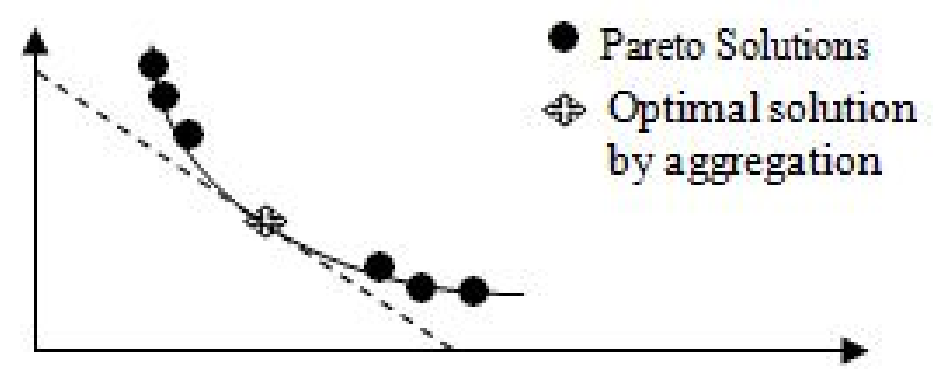

Figure 8:

\section{Multi-objective optimization and computing of lower bounds}

The computing of lower bounds has been studied in literature for several scheduling problems which we quote: the problems with a machine [27], with parallel machines [28] and [29], the hybrid flow-shop problems [30] and the flexible job shop problems [31]. These proposed methods for computing of the lower bounds are generally based on the relaxation of constraints (preemption of tasks, constraints related resources ...) to minimize one or more criteria for optimal scheduling. Based on these methods and seen that we don't have information on the optimal solutions associated with different cost functions $f_{i}$ for our problem we should compute the minimum value to determine the scaling constants $c_{i}$. For this objective, we use the relaxation of various constraints. To find a minimum value associated with the criterion of total travel cost $f_{1}=$ $\sum_{k \in K} \sum_{i \in N} \sum_{j \in N} C_{k} d_{i j} X_{i j k}$, we have treated this problem to the travelling salesman problem when we try to minimize $\sum_{i \in N} \sum_{j \in N} d_{i j} X_{i j}$. We subsequently determine the routing crossed by a single vehicle, minimizing the total travel distance by incorporating the constraints and capacity precedence. What gives us $d_{\min . p . c}$. 


$$
d_{\min . p . c}=\min \left(\sum_{i \in N} \sum_{j \in N} d_{i j} X_{i j}\right)
$$

By setting $K$, the number of vehicles used, we get:

$$
d_{\min k . p . c}=\frac{d_{\min . p . c}}{K}
$$

Consequently, we acquire a value $f_{1 b}$ that represents a minimum value of the total travel cost.

$$
f_{1 b}=\sum_{k \in K} C_{k} d_{\min k . p . c}
$$

To determine the minimum value of the total tardiness time, we fix $\mathrm{K}$ the vehicles number and find out the population $\mathrm{P}_{\text {node/vehicule }}$. Thus, we calculate the total tardiness time for each individual and determine the minimum.

$$
f_{2 b}=\sum_{k \in K} \sum_{i \in N} \sum_{j \in N} \max \left(0, D_{i}-l_{i}\right) X_{i j k}
$$

Knowing that for the criterion of tardiness, a better lower bound is zero. We will have therefore:

$$
\left\{\begin{array}{l}
c_{2}=\frac{1}{f_{2 b}} \\
\text { s.c. } f_{2 b} \neq 0
\end{array}\right.
$$

\subsection{Computational results}

To test our approach, we use benchmark problem instances generated by Li and Lim [19] from Solomon's ones [32]. Corresponding to Solomon's classification of C1, C2, R1, R2, RC1 and $\mathrm{RC} 2$, their data sets were also generated in six classes: LC1, LC2, LR1, LR2, LRC1 and LRC2. The LC problems are clustered whereas in the LR problems, providers and customers are randomly generated. Therefore in the LRC problems the providers and the customers are partially clustered and partially randomly distributed. While LC1, LR1 and LRC1 problems have a short scheduling horizon, LC2, LR2 and LRC2 have longer scheduling one. [33] In our work, we consider a vehicle number $\mathrm{k}$ ranging between 1 and 25. Table 1 shows the results of our simulation using the parameters of the problem LRC1. Of course, for every given solution, we note the corresponding routing, crossed by each vehicle.

$N_{\text {sol }}$ : represents the number of non dominated solutions.

$N_{k}$ : represents the vehicles number used.

We observe that our approach generates a multiple number of solutions that give flexibility of choice for the decision maker and that by using two different methods to determine the vehicles number used, minimizing the compromise between the total travel cost and the total tardiness time. We also observe that we obtain a total tardiness equal to zero with a tolerable cost.

\section{Conclusion}

In this paper, we have presented our approach to solve the m-PDPTW, based on Pareto dominance method, with use of genetic algorithm and lower bounds. Our purpose was in a first part a brief literature review on the VRP, 1-PDPTW and m-PDPTW. The mathematical formulation of our problem is detailed in second part. Then, we have detailed the use aggregation 


\begin{tabular}{|c|c|c|c|c|c|c|c|}
\hline LRC1 & $N_{\text {sol }}$ & $N_{k}$ & $f_{1 b}$ & $f_{2 b}$ & $f_{1}$ & $f_{2}$ & $F(x)$ \\
\hline LRC101 & 4 & 11 & 67971,97 & 39,08 & 187085,73 & 2,173 & 1,09 \\
& & & & & 192150,78 & 2,09 & \\
& & & & & 192843,93 & 1,32 & \\
& & & & & 194911,78 & 0,651 & \\
\hline LRC101 & 1 & 25 & 67971,97 & 0 & 216261,26 & 0 & 2,49 \\
\hline LRC103 & 2 & 11 & 645,1144 & 0 & 1946,2465 & 0 & 1,09 \\
& & & & & 1711,304 & 0,429 & \\
\hline LRC103 & 1 & 25 & 645,1144 & 0 & 2081,1978 & 0 & 2,49 \\
\hline LRC105 & 3 & 9 & 697,0005 & 0,823 & 1862,0582 & 4,28 & 0,89 \\
& & & & & 1965,2465 & 0,823 & \\
& & & & & 1947,6537 & 4,27 & \\
\hline LRC105 & 2 & 25 & 697,0005 & 0 & 2171,1193 & 0,107 & 2,49 \\
& & & & & 2246,6729 & 0 & \\
\hline LRC107 & 1 & 11 & 647,345 & 0 & 1803,9515 & 0 & 1,09 \\
\hline LRC107 & 1 & 25 & 647,345 & 0 & 2171,1006 & 0 & 2,49 \\
\hline
\end{tabular}

Table 1: Results for the LRC1 problem

method and lower bounds to determine a set of solutions, minimizing our objective functions. Simulation was presented in a last part by using benchmark's data.

\section{Bibliography}

[1] H. Ezzedine T. Bonte C. Kolski and C. Tahon. Integration of traffic management and traveller information systems: basic principles and case study in intermodal transport system management. International Journal of Computers, Communications \& Control (IJCCC), ISSN 1841 9836, E-ISSN 1841-9844 Vol. III, No. 3, pp. 281-294, 2008.

[2] Christofides N. Mingozzi A. and Toth P. The vehicle routing problem. In Combinatorial Optimization, volume 11, pages 315-338. John Wiley, 1979.

[3] Lenstra J. and Rinnooy Kan A. Complexity of the vehicle routing and scheduling problems. In Networks, volume 11, pages 221-228. Springer, 1981.

[4] Nabaa M. Zeddini B. and Tranouez P. Approche décentralisée pour résoudre le problème du transport ŕ la demande. Schedae, prépublication $n^{\circ} 23$, (fascicule $n^{\circ} 2$, p. 133-140), 2007.

[5] Toth P. and Vigo D. The vehicle routing problem, SIAM Monographs on Discrete Mathematics and Applications, 2002, ISBN 0-89871-498-2.

[6] Montamenni R. Gambardella L.M. Rizzoli A.E. and Donati A.V. A new algorithm for a Dynamic Vehicle Routing Problem based on Ant Colony System. IDSIA, Switzerland, 2002.

[7] Sorin C. Negulescu, Claudiu V. Kifor, and Constantin Oprean. Ant Colony Solving Multiple Constraints Problem: Vehicle Route Allocation. Int. J. of Computers, Communications \& Control (IJCCC), ISSN 1841-9836, E-ISSN 1841-9844, Vol. III, No. 4, pp. 366-373, 2008. 
[8] Kaoru Hirota, Fangyan Dong and Kewei Chen. A Computational Intelligence Approach to VRSDP (Vehicle Routing, Scheduling, and Dispatching Problems). ICCCC, Baile FelixOradea, Romania pp. 53-54, 2006.

[9] Savelsbergh M.P.W. and SOL M., "The general pickup and delivery problem", Transportation Science 1995.

[10] Psaraftis H.N. An exact algorithm for the single vehicle many to many immediate request dial a ride problem with time windows. Transportation Science, 17, 351-357, 1983.

[11] Psaraftis H.N. A dynamic programming solution to the single vehicle many to many immediate request dial a ride problem. Transportation Science, 14(2):130-154, 1980.

[12] Jih. W. and Hsu J. Dynamic vehicle routing using hybrid genetic algorithms. International Conference on Robotics and Automation, pages 453-458, 1999.

[13] Velasco N. Dejax P. Gueret C. and Prins C. Un algorithme génétique pour un problème de collecte bi-objectif. MOSIM 2006.

[14] Kammarti. R. Hammadi. S. Borne. P. and Ksouri. M. A new hybrid evolutionary approach for the pickup and delivery problem with time windows. IEEE International Conference on Systems, Man and Cybernetic. 2004. Volume 2, P 1498-1503, Oct 2004.

[15] Kammarti. R. Hammadi. S. Borne. P. and Ksouri. M. "Improved tabu search in an hybrid evolutionary approach for the pickup and delivery problem with time windows", Intelligent Transportation Systems, 2005. Proceeding 2005 IEEE, p 148-153, 2005.

[16] Kammarti. R. Hammadi. S. Borne P. and Ksouri. M. "Solving the Real Time dynamic Pickup and Delivery Problem with an hybrid evolutionary approch". Multiconference on Computational Engineering in Systems Application. Volume 2, P 1520-1525, Oct 2006.

[17] Sol M. and Savelsbergh M. A branch-and-price algorithm for the pickup and delivery problem with time windows. Memorandum COSOR 94-22, Dept. of Mathematics and Computing Science, Eindoven University of Technology, Eindoven, The Netherlands, 1994.

[18] Quan L. and Maged M. A new insertion-based construction heuristic for solving the pickup and delivery problem with time windows. Science direct, European Journal of Operational Research 2003.

[19] Li H. and Lim A. A metaheuristic for the pickup and delivery problem with time windows. In IEEE International Conference on Tools with Artificial Intelligence, volume 13, pages 160167, 2001.

[20] Li H. Lim A. and Rodrigues B.. Solving the pickup and delivery problem with time windows using squeaky wheel" optimization with local search. SMU Business Conference Paper Series, 2002 .

[21] Harbaoui Dridi I. Kammarti R. Borne P. and Ksouri M. Un Algorithme génétique pour le problème de ramassage et de livraison avec fenętres de temps ŕ plusieurs véhicules. CIFA 2008, Bucarest (Roumanie), Septembre 2008 Proc. Article 176.

[22] Harbaoui Dridi I. Kammarti R. Borne P. and Ksouri M. Approche multicritère pour le problème de ramassage et de livraison avec fenętres de temps ŕ plusieurs véhicules. Symposium LT'2009, Tunisie, (Sousse), LT09-006, Mars 2009. 
[23] Harbaoui Dridi I. Kammarti R. Borne P. and Ksouri M. Genetic Algorithm for Multicriteria Optimization of a Multi-Pickup and Delivery Problem with Time Windows. 13th INCOM IFAC symposium, Moscow (Russia), pp 1521-1526, June 2009.

[24] Harbaoui Dridi I. Kammarti R. Ksouri M and Borne P. A Genetic Algorithm for the MultiPickup and Delivery Problem with time windows. Studies in Informatics and Control (SIC), Vol 18, N², pp 173-180, Juin 2009.

[25] Pareto. V. Cours d'économie politique, Lausanne : Rouge, 1896-7, reproduit in Vilfredo Pareto, Oeuvres complètes, Genève : Librairie Droz, 1964.

[26] Hwang, C. and Masud, A. Multiple objective decision making - methods and applications. In Lectures Notes in Economics and Mathematical Systems, volume 164. Springer-Verlag, Berlin, 1979.

[27] Jouglet. A, Baptiste. B et Carlier. J. Exact Procedures for Single Machine Total cost Scheduling. Proceeding of the IEEE / SMC'02 Conf. 6-9 October, Hammamet, Tunisia, 2002.

[28] Jurich, B. Scheduling Jobs in Shops with Multi-purpose Machines. Ph.D thesis, Fachbereich Mathematik / Informatik, Universitat Osnabruck., 1992.

[29] Carlier, J. Scheduling jobs with release dates and tails on identical machines to minimize the makespan. European Journal of Operational Research , 29 (1987) 298-306, North-Holland.

[30] Billaut, J.C, Carlier, J, Néron, E. Ordonnancement d'ateliers ŕ ressources multiples. Chapitre dans l'ouvrage : Ordonnancement de la production, Edition Hermès, France, 2001.

[31] I. Kacem. Ordonnancement multicritères des job shops flexibles : formulation, bornes inferieures et approche éévolutionniste coopérative. Thèse en Automatique et informatique industrielle ŕ l'université de Lille 1, 2003.

[32] Solomon M.M., "Algorithms for the vehicle Routing and Scheduling Problem with Time Window Constraints", Operations Research, 41, 469-488, 1987.

[33] Kammarti. R. Hammadi. S. Borne. P. and Ksouri. M. "Lower Bounds In An Hybrid Evolutionary Approach For The Pickup And Delivery Problem With Time Windows". IEEE International Conference on Systems, Man and Cybernetics. 2005. Volume 2, P 1156-1161, Oct 2005. 